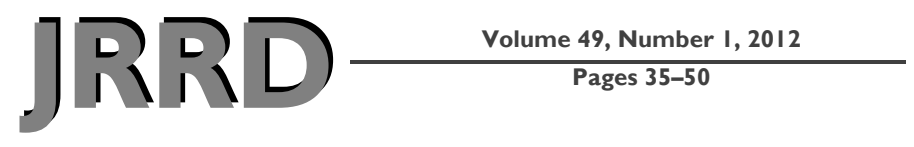

\title{
Clinical evaluation of semiautonomous smart wheelchair architecture (Drive-Safe System) with visually impaired individuals
}

\author{
Vinod Sharma, PhD $;^{1 *}$ Richard C. Simpson, PhD, ATP; ${ }^{1-3}$ Edmund F. LoPresti, PhD; ${ }^{4}$ Mark Schmeler, OTR/L, ATP ${ }^{2}$ \\ Departments of ${ }^{1}$ Bioengineering and ${ }^{2}$ Rehabilitation Science and Technology, University of Pittsburgh, Pittsburgh, \\ PA; ${ }^{3}$ Human Engineering Research Laboratories, Department of Veterans Affairs Pittsburgh Healthcare System, Pitts- \\ burgh, PA; ${ }^{4}$ AT Sciences, Pittsburgh, PA
}

\begin{abstract}
Nonambulatory, visually impaired individuals mostly rely on caregivers for their day-to-day mobility needs. The Drive-Safe System (DSS) is a modular, semiautonomous smart wheelchair system aimed at providing independent mobility to people with visual and mobility impairments. In this project, clinical evaluation of the DSS was performed in a controlled laboratory setting with individuals who have visual impairment but no mobility impairment. Their performance using DSS was compared with their performance using a standard cane for navigation assistance. Participants rated their subjective appraisal of the DSS by using the National Aeronautics and Space Administration-Task Load Index inventory. DSS significantly reduced the number and severity of collisions compared with using a cane alone and without increasing the time required to complete the task. Users rated DSS favorably; they experienced less physical demand when using the DSS, but did not feel any difference in perceived effort, mental demand, and level of frustration when using the DSS alone or along with a cane in comparison with using a cane alone. These findings suggest that the DSS can be a safe, reliable, and easy-to-learn and operate independent mobility solution for visually impaired wheelchair users.
\end{abstract}

Key words: artificial intelligence, distributed systems, embedded systems, human-robot interaction, intelligent mobility aids, power wheelchairs, rehabilitation, robotics, sensors, shared control, smart wheelchair.

\section{INTRODUCTION}

\section{Problem Statement}

Loss of independent mobility can alter a person's productivity, personal freedom, and fulfillment and give a feeling of confinement and dependence [1]. It can also adversely affect a person's psychosocial and emotional health and limit his or her educational and vocational opportunities. Physical impairment when combined with visual impairment furthers the loss of independent mobility [1-3]. In aging populations, physical impairment is often accompanied by visual impairment due to causes such as macular degeneration, cataracts, glaucoma, and diabetic retinopathy [1]. Nonambulatory, visually impaired individuals often rely on their caregivers for their

\footnotetext{
Abbreviations: ADL $=$ activity of daily living, DSS $=$ DriveSafe System, FEW = Functioning Everyday with a Wheelchair, NASA-TLX = National Aeronautics and Space AdministrationTask Load Index, NCT = number of collisions per trial, NCT-I = NCT-Type I, NCT-II = NCT-Type II, NCT-III = NCT-Type III, NCT-T $=$ NCT-total, $\mathrm{SD}=$ standard deviation, TCT $=$ task completion time, TLX-E = NASA-TLX Effort, TLX-F = NASATLX Frustration, TLX-MD = NASA-TLX Mental Demand, TLX-PD = NASA-TLX Physical Demand, TLX-TWL = NASA-TLX Total Workload, UR = ultrasonic rangefinders.

*Address all correspondence to Vinod Sharma, PhD; Suite 224, Loeffler Building, 121 Meyran Ave, Pittsburgh, PA 15213; 412-953-2157. Email: vks3@pitt.edu

http://dx.doi.org/10.1682/JRRD.2010.03.0022
} 
activities of daily living (ADLs), which can lower their self-esteem, creating feelings of worthlessness and learned helplessness and thus affecting their psychological wellbeing in addition to their quality of life [1].

"The 2007 disability status report [4] shows that 40.3 percent of older adults aged 75 and above who are living at home have conditions that substantially limit one or more basic physical activities such as walking, climbing stairs, reaching, lifting, or carrying. A further 3.6 percent of older adults in this population have sensory disabilities, which include blindness or severe visual impairment. Whereas in 1990, only 2.74 percent of older adults age 65 and above used wheelchairs, in 1990, their percentage grew to 5.2 percent by 2005. Among noninstitutionalized adults aged 85 and above, 12.28 percent use wheelchairs [5], most of which are manual wheelchairs pushed by a caregiver or family member.”a

Currently, the majority of nonambulatory, visually impaired persons use wheelchairs that are pushed by their caregivers [6-7]. There are reports of people using canes for navigation assistance with manual wheelchairs [8-9]. In this scenario, the user holds the cane on his or her lap and pushes the wheelchair and then stops and scans the environment for obstacles with the cane. The issue with this approach is that it requires a lot of effort from users to push the wheelchair while holding the cane in an awkward position on their lap. Individuals with physical impairments often have low stamina and strength and are more likely to be obese, which make pushing the wheelchair extremely difficult [10]. There are also reports of individuals with visual impairment using a guide dog for navigation assistance with a manual wheelchair [8]. The guide dog leads the manual wheelchair and the user pushes the wheelchair to follow the guide dog. This approach is less cumbersome than using a cane but still requires significant physical effort to push the wheelchair, which discourages people from using this option for independent mobility.

Powered mobility is considered the ideal choice for individuals with low physical stamina because they can conserve their energy for use in their ADLs without having to rely on others for their mobility needs $[7,11]$. Nonambulatory, visually impaired people often lack the cognitive, visual, and motor skills required to safely maneuver a power wheelchair and are therefore often denied powered mobility as a result of concerns regarding the safety of the user and the environment $[10,12]$. Researchers from engineering and the clinical side have attempted to make powered mobility available to such populations without compromising the safety of the user and the environment [3,10,13-14].

For example, researchers have evaluated the effectiveness of using a cane with a power wheelchair. They found that training required significant time and resources and often required modification to users' living spaces $[9,14]$. Researchers have tried to teach and evaluate the effectiveness of using a guide dog or service dog as a navigation assistant with a power wheelchair. While a few published case studies using this approach have been published in the literature, none led to a commercially viable product [15].

In recent years, the reduced cost and size of computing devices and the advancement in sensor technologies have attracted researchers from robotics to incorporate these technologies in the design of smart wheelchairs. Smart wheelchairs are designed to accommodate individuals with severe disabilities who cannot operate existing power wheelchairs [13]. In detailed reviews of smart wheelchair technologies [13] and their target populations [16], Simpson et al. present a segment of users who will benefit from the use of smart wheelchairs.

However, even after more than three decades of research in powered mobility, very few smart wheelchairs are commercially available in the United States. For example, the Wheelchair Pathfinder, a commercial electronic mobility aid sold by Nurion Industries, has been discontinued [17]. Smart wheelchairs have found limited success in Europe as well. For example, the CALL Center smart power wheelchair sold in Europe by Smile Rehab, Ltd (Berkshire, United Kingdom) includes bump sensors, sonar sensors, and the ability to follow tape tracks on the floor [18]. This smart wheelchair was targeted at teaching powered mobility skills to children and was never meant to provide safe navigation practices for long-term wheelchair use [19].

\section{Drive-Safe System}

Our research group has been involved with the design, development, and evaluation of smart wheelchairs for nonambulatory, visually impaired individuals for the past 15 years [18-23]. "The Drive-Safe System (DSS) is an add-on, distributed, shared control navigation assistance system for power wheelchairs designed to provide safe and independent mobility to people with visual and mobility impairments (Figure 1). DSS allows users to choose the destinations, plan their paths, and perform 


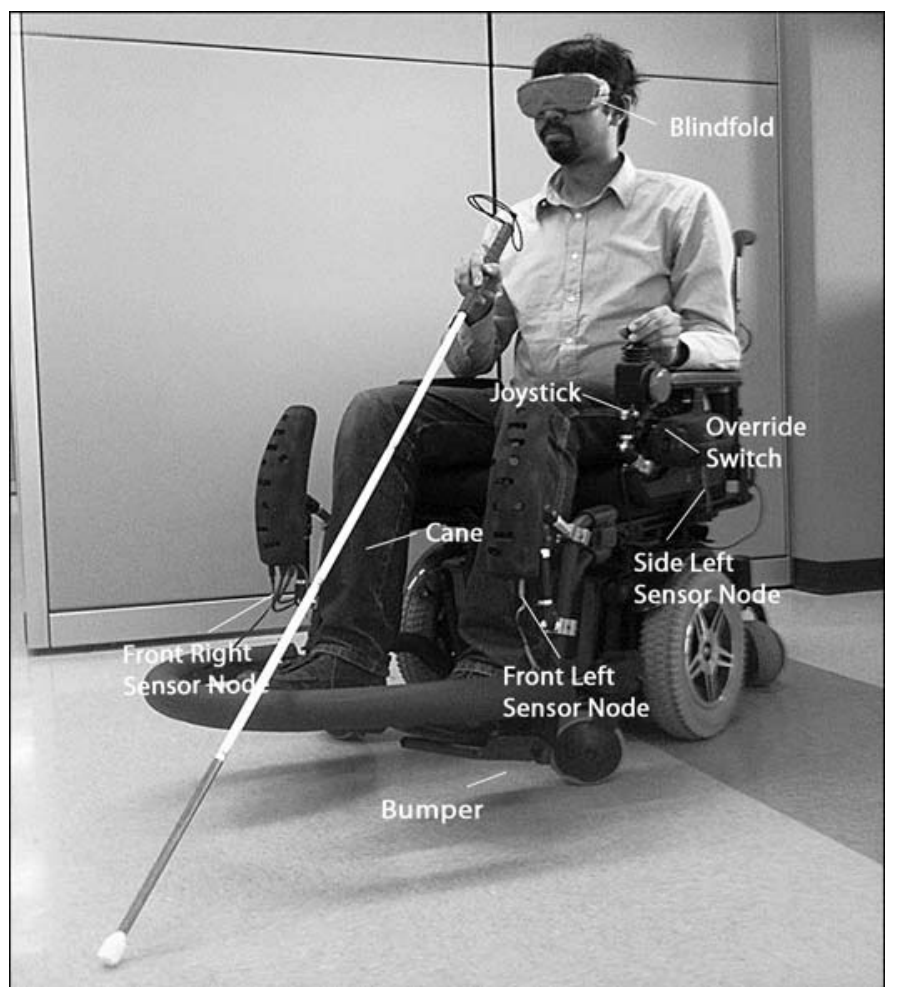

Figure 1.

Drive-Safe System (DSS).

some navigation actions, while automatically overriding unsafe maneuvers through autonomous collision avoidance, automatic wall following, and door crossing."” The DSS is fifth-generation smart wheelchair architecture for addressing the issues and concerns raised by participants, clinicians, wheelchair manufacturers, distributors, and researchers with our previous smart wheelchair prototypes. The distributed, modular architecture of the DSS can be retrofitted to most power wheelchairs commercially available in the United States. Additionally, whenever individuals change their wheelchair, the DSS architecture from their old wheelchair can be mounted on their new wheelchair.

The architecture of the DSS contains hardware and software. The two main parts of the DSS hardware architecture are the sensor nodes and a translator node. Five sensor nodes can be mounted by quick-release mounting mechanisms on the front left, front right, side left, side right, and rear side of the wheelchair. Each sensor node contains 10 proximity sensors (5 ultrasound sensors and 5 infrared sensors) and two touch-sensitive bumpers.
Proximity sensors in sensor nodes are directed such that they provide coverage around the wheelchair and can detect obstacles as low as $3 \mathrm{in}$. from ground level to overhanging obstacles as high as 60 in. from ground level. Sensor nodes also provide users with auditory feedback about the position of an obstacle so that they can change their trajectory to avoid collision.

The translator node acts as the brain of the DSS architecture and collects information about obstacle positions from all five sensor nodes and calculates the obstacle in the trajectory of wheelchair movement based on the users intended direction of movement. The presence of obstacles in the wheelchair's trajectory will either slow down or stop the wheelchair. The DSS obstacleavoidance algorithm incorporates the range data from proximity sensors and acquires the user's intended direction of movement from the joystick. If an obstacle is in the trajectory of the wheelchair's movement, the DSS will slow down the wheelchair and, at a certain distance from the obstacle, will stop the wheelchair to avoid potential collision.

A detailed description of the DSS hardware, software, and engineering and performance evaluation is published in LoPresti et al. [24]. In the present study, we evaluate the effectiveness of the DSS in providing safe independent mobility to people with visual impairments but no mobility impairments in controlled laboratory settings. We also compare the navigation performance of these individuals with the DSS to the conventional navigation assistance approach of using a cane.

\section{METHODS}

\section{Participants}

This study used people with visual impairment but no mobility impairment. People with visual impairment were employed to evaluate the merits of the DSS for people with both visual and mobility impairments because both populations have similar skill sets of cane usage, sound localization, and geographic reasoning [25]. Further, both experience similar challenges in performing ADLs. Seven participants with visual impairment (three females, four males) were recruited for this study. The mean age of participants was 53.71 years (standard deviation $[\mathrm{SD}]=16.41$ years). Five participants had prior experience with wheeled mobility devices (walkers and manual wheelchairs) for short periods of time, mostly 
when they were unable to walk because of a temporary medical condition (e.g., fractured bone, surgery). None of the participants was using a wheeled mobility device at the time that they participated in the study.

\section{Instrumentation}

“A Quantum-600 midwheel drive power wheelchair, manufactured by Pride Mobility Products [Exeter, Pennsylvania] equipped with the DSS (Figure 1) was used for this study. The wheelchair was controlled by a proportional joystick. The maximum forward and reverse speeds of the wheelchair were set to $1.7 \mathrm{mph}$ and $1.3 \mathrm{mph}$, respectively, to closely match the average driving speed for wheelchair users [26]." "a The study was conducted in a trial area measuring $3.30 \times 7.50 \mathrm{~m}^{2}$. Cylindrical cardboard tubes 8 in. $(20 \mathrm{~cm})$ in diameter and 60 in. $(152.4 \mathrm{~cm})$ in height were used as obstacles, and wooden benches 36 in. $(91.44 \mathrm{~cm})$ in height were used to mark the boundaries of the trial area.

The obstacles in the obstacle courses were placed in such a way that the level of difficulty presented to the participants would be the same in each obstacle course. There were seven obstacles in each of the six obstacle courses. The placement of these seven obstacles and the positions of the participant in relation to them were designed to make it hard for participants to navigate based on past trials so that learning effects could be effectively mitigated. Even though there were no physical doors or walls in the experimental set up, obstacles were placed so as to mimic door situations and the width between two obstacles was kept between 4 and $5 \mathrm{ft}$. The obstacles triggered the door-crossing mode in the DSS architecture, which facilitates the crossing of narrow openings. Side walls defining the boundary of the obstacle course triggered the wall-following mode in the DSS, which helped participants travel close to the walls without getting stopped repeatedly.

Six obstacle courses were used in this study, and in every course, participants were expected to face head-on obstacles; the only way to avoid the obstacles was to make sharp left or sharp right turns. Even after the aforementioned provisions, measuring whether each obstacle course presented an equal level of difficulty to participants was difficult. The difficulty of the obstacle courses was found to be very subjective to the participants' driving habits and their ability to use navigation assistance in three experimental conditions.

\section{Pretraining}

Before participating in the study, the investigator read and explained each section of the informed consent form to each participant. Once each participant indicated that they understood and agreed to participate, they signed the informed consent form. A copy of the informed consent form was given to the participants upon completion of the experiment.

Depending on each participant's requirements, the seating and positioning of the wheelchair were adjusted by the investigator. "For example, the wheelchair joystick was mounted on the right or the left side of the wheelchair, depending upon the participant's dominant hand. Backrest and footrests were adjusted such that participants could sit comfortably on the wheelchair." Because none of the recruited participants had prior experience using a power wheelchair, the investigator explained to them the functionality of the joystick and maneuvering of the wheelchair using the joystick.

\section{Training}

Three experimental driving conditions were evaluated in this study: DSS, Cane, and DSS + Cane. To equate the effect of training, we provided an equal amount of training in all three experimental conditions. Training in each condition took 15 to 20 minutes. Since none of the participants had used a power wheelchair before the study, driving the wheelchair in all three conditions was equally novel for them. Participants were required to use their dominant hand for driving and the nondominant hand for scanning. The protocol for training in this study was the same as that used in our earlier study with nondisabled individuals [27]. A detailed description of the training protocol is provided in Sharma et al. [27].

\section{Experimental Protocol}

The experimental protocol used in this study was similar to the protocol in our prior study of DSS with nondisabled individuals, as reported in Sharma et al. [27]. Three experimental driving conditions-Cane, DSS, and DSS + Cane-were used in this study and participants completed six trials in each of three experimental conditions (Figure 2). Six obstacle courses (Figure 3) were used for in this study and the order of these obstacle courses in each experimental condition was randomly selected. In the Cane condition, participants used a 48 in. long white cane for navigation assistance while using a power wheelchair. In the DSS condition, navigation assistance was solely pro- 


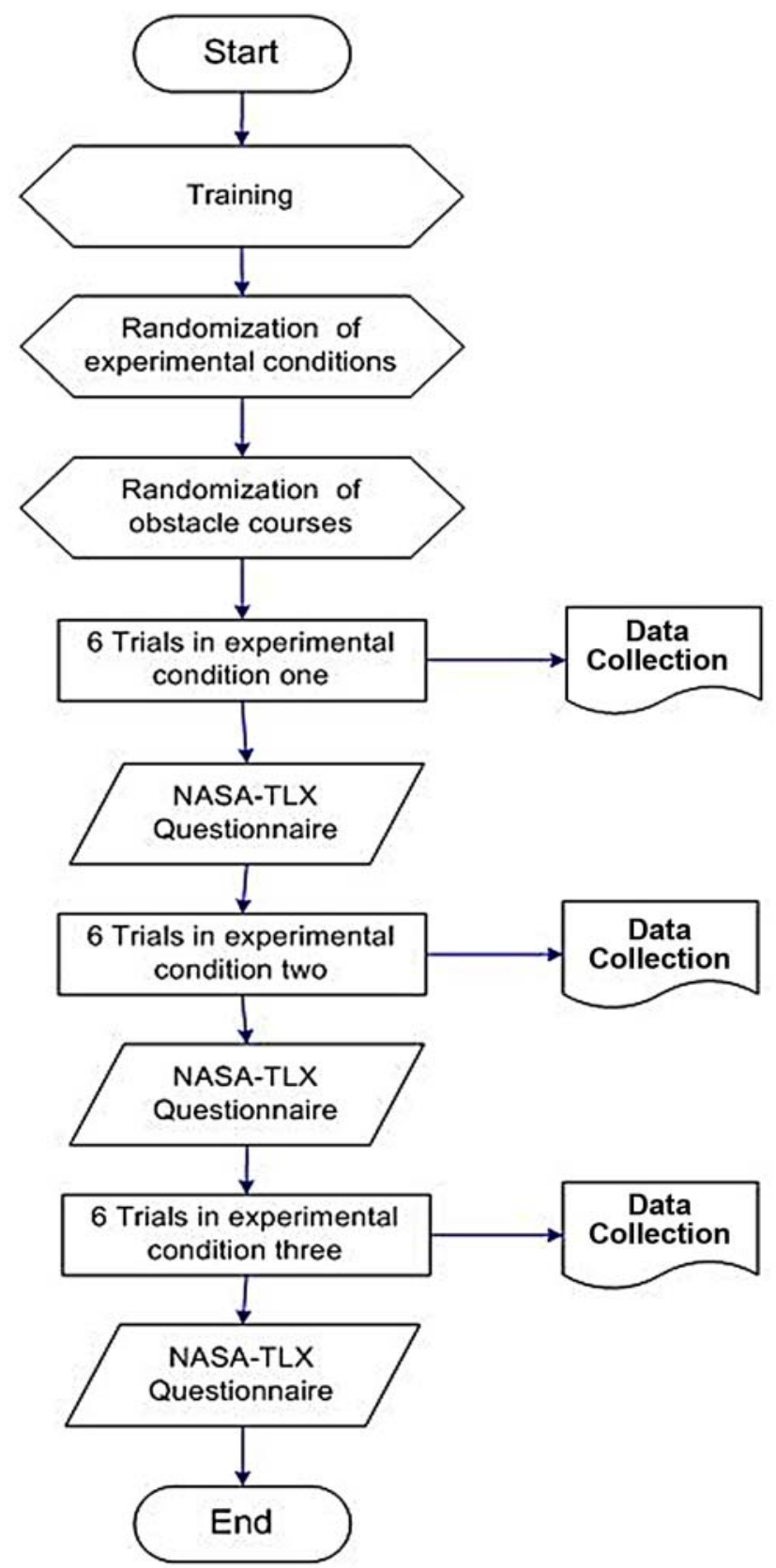

Figure 2.

Study protocol. NASA-TLX = National Aeronautics and Space Administration-Task Load Index.

vided by the DSS. Finally, in the DSS + Cane condition, navigation assistance was provided by both the DSS and the cane. After each experimental condition, participants rated their subjective experiences by using the National Aeronautics and Space Administration-Task Load Index (NASA-TLX) inventory [28].
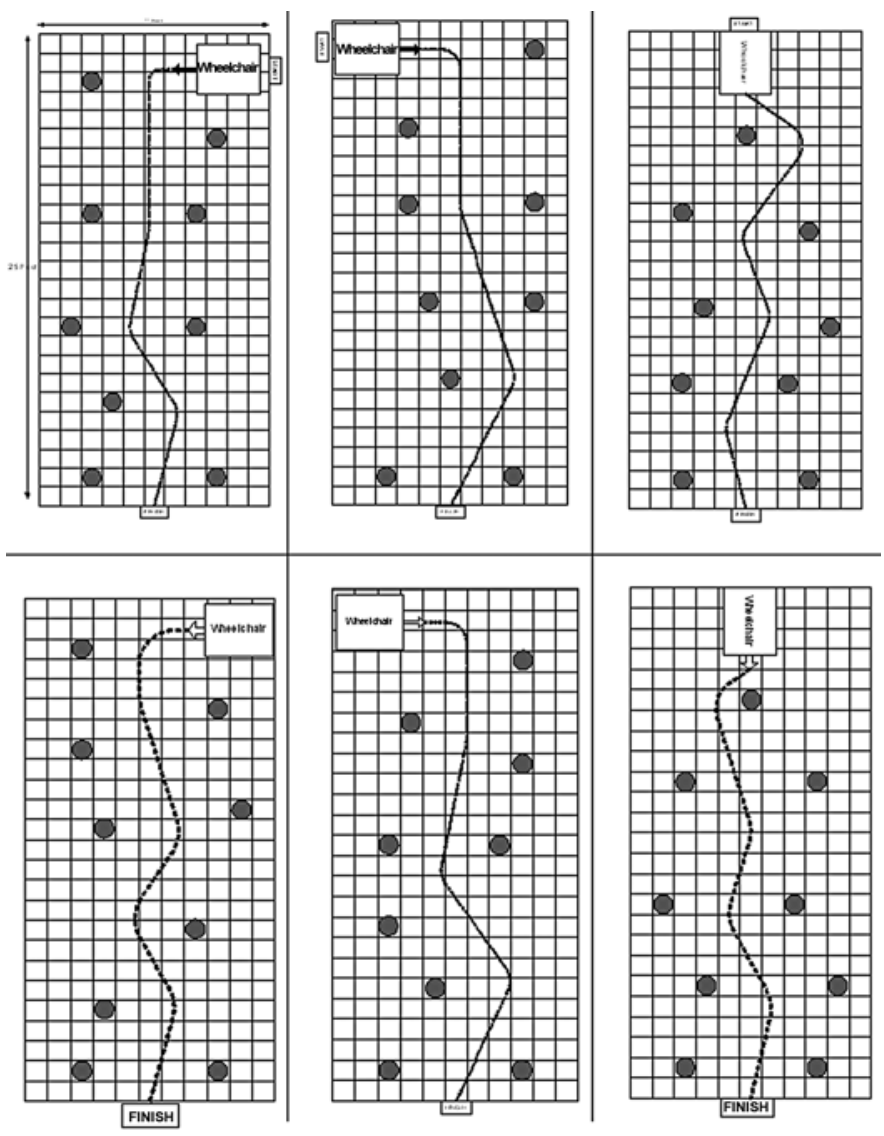

Figure 3.

Obstacle courses.

\section{Dependent Variables}

Dependent variables used in this study were both objective and subjective in nature. Objective measures were task completion time (TCT) and number of collisions per trial (NCT). Measures for collisions were further categorized according to the severity of collisions (Figure 4). Type I were the least severe collisions while Type III were of highest severity. The self-reported, survey-based NASA-TLX subjective workload index along with its four subscales (Mental Demand [TLX-MD], Physical Demand [TLX-PD], Effort [TLX-E], and Frustration [TLX-F]) were used as subjective dependent variables [28-30].

The multidimensional nature of the NASA-TLX allowed us to evaluate the task independent workload associated with the use of the DSS and the workload associated with the interaction between the task and the DSS. Additionally, the NASA-TLX has proven reliability and validity and has been used extensively in various 


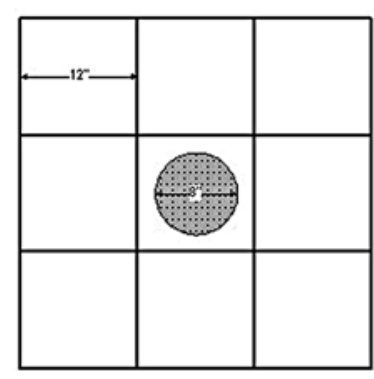

Obstacle Placement

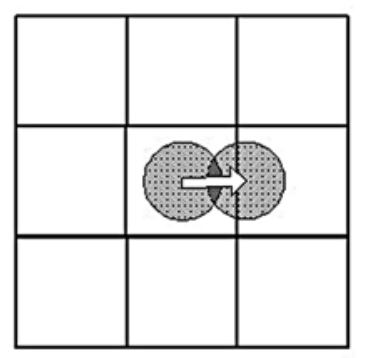

Type II Collision

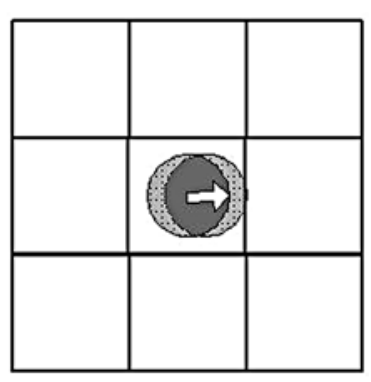

Type I Collision

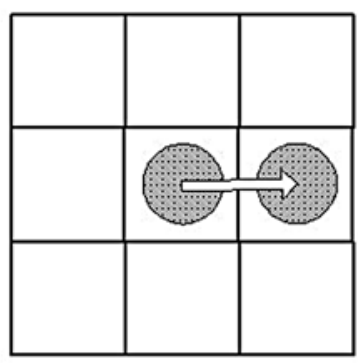

Type III Collision
Figure 4.

Collision types.

fields of research around the world [28]. The NASATLX was quick and easy to administer and was completed by the participant, leaving no room for experimenter bias.

Another advantage of using the NASA-TLX was that it did not require long-term use of the DSS, as opposed to other outcome measure tools such as The Quebec User Evaluation of Satisfaction with Assistive Technology [31], the Matching Person \& Technology assessment [32], and Functioning Everyday with a Wheelchair (FEW) [33-34], which require long-term use of an assistive technology device and need to be administered by certified clinicians.

In the NASA-TLX, TLX-MD is defined as the mental and perceptual effort (e.g., thinking, deciding, calculating, remembering, searching, maneuvering) required to finish a navigation task. TLX-PD is defined as the physical activity required (e.g., scanning, pushing, pulling the cane, maneuvering the joystick, turning, controlling, activating) to finish the navigation task. Further, TLX-PD also represents whether the task was easy or demanding, slow or quick, restful or laborious. Temporal demand also considers the pace of the task progression, such as slow and leisurely or rapid and frantic. TLX-E is defined as the amount of work (both mental and physical) participants had to exert to achieve their level of performance. Mental effort and physical effort are different from mental demand and physical demand. Demand is the load associated with the task, while effort is the load associated with the interaction between the user and the task. TLX-F measures the extent to which a person feels insecure, discouraged, irritated, stressed, and annoyed versus secure, gratified, content, relaxed, and complacent during the task. Two other items in the NASA-TLX, viz. temporal demand and performance, were not relevant to the objectives of this study and hence will not be reported here. The total workload (TLX-TWL) was calculated from the sum of all the individual subscales.

\section{Specific Aims and Hypotheses}

The primary purpose of this study was to determine the comparative effectiveness of the DSS and cane in providing navigation assistance with a power wheelchair to people with visual impairment. Another aim was to evaluate the subjective workload associated with use of the DSS alone and the DSS along with a cane on navigation tasks and compare it with the subjective workload associated with the use of a cane alone on similar navigation tasks. We hypothesized that users would have fewer collisions when using the DSS but that TCT would not differ. We also hypothesized that participants would experience less subjective workload when using the DSS than when using the cane alone.

\section{Data Analysis}

"Normality of each dependent variable was tested using the Shapiro-Wilk test. A general linear model repeated measures analysis of variance was used for analyses of normally distributed dependent variables with the significance level set at $p<0.05$. Pair-wise comparisons were performed using a standard $t$-test with a Bonferroni adjustment for multiple comparisons. Table 1 describes the statistical notations used in this article. Data that were not normally distributed were analyzed using nonparametric tests for related samples. Friedman's test was used to compare the underlying distributions across all three experimental conditions with significance level set at $p<$ 0.05. Pairwise comparisons between conditions were performed using the Wilcoxon Signed Ranks test with a significance level set at $p<0.05$.” 
Table 1.

Statistical symbols used.

\begin{tabular}{cl}
\hline Symbol & \multicolumn{1}{c}{ Meaning } \\
\hline$Z$ & Standard normal variable \\
$p$ & Attained level of significance \\
$\chi^{2}$ & Chi-square distribution \\
$F$ & $F$-distribution variable \\
$\mu$ & Arithmetic mean \\
$\sigma$ & Standard deviation \\
\hline \hline
\end{tabular}

\section{RESULTS}

\section{Collisions}

Figure 5 shows the occurrence of collisions across the three experimental conditions. Collisions were only those instances in which the wheelchair hit/displaced the obstacles. In several instances, participants hit an obstacle with the cane while scanning, but these hits were not counted as collisions. There were also a few instances when obstacles moved a few inches (less than 6 in.) because of the hit from the cane, but these were also not counted as collisions. In the following subsections, we will discuss the performance of the three conditions for the various types of collisions.

\section{Type I Collisions}

Table 2 shows the mean \pm SD Type I Collisions (NCT-I) for the three conditions. A Shapiro-Wilk test showed that NCT-I was not normally distributed. The difference between the DSS condition and the Cane condition on NCT-I was not statistically significant $(Z=1.725$, $p=0.08$ ). Similarly, the difference between the DSS condition and the DSS + Cane condition was not statistically significant $(Z=1.342, p=0.18)$. However, the mean NCT-I was significantly lower for the DSS + Cane condition than the Cane condition $(Z=2.546, p=0.01)$.

\section{Type II Collisions}

Table 2 shows the mean \pm SD Type II Collisions (NCT-II) for the Cane and DSS + Cane conditions. No Type II collisions occurred with the DSS. A ShapiroWilk test showed that NCT-II was not normally distributed. The Cane condition had significantly greater NCTII than the DSS condition ( $Z=2.375, p=0.02$ ) and the DSS + Cane condition $(Z=2.388, p=0.02)$. NCT-II did not significantly differ between the DSS and DSS + Cane conditions $(Z=-1.00, p=0.32)$.

\section{Type III Collisions}

Table 2 shows the mean \pm SD Type III Collisions (NCT-III) for the Cane condition. No NCT-III occurred for either the DSS or DSS + Cane conditions. A Shapiro-Wilk test showed that NCT-III was not normally distributed. Participants had significantly greater NCT-III in the Cane condition than in both the DSS + Cane condition $(Z=2.201$, $p=0.03)$ and the DSS condition $(Z=2.201, p=0.03)$.

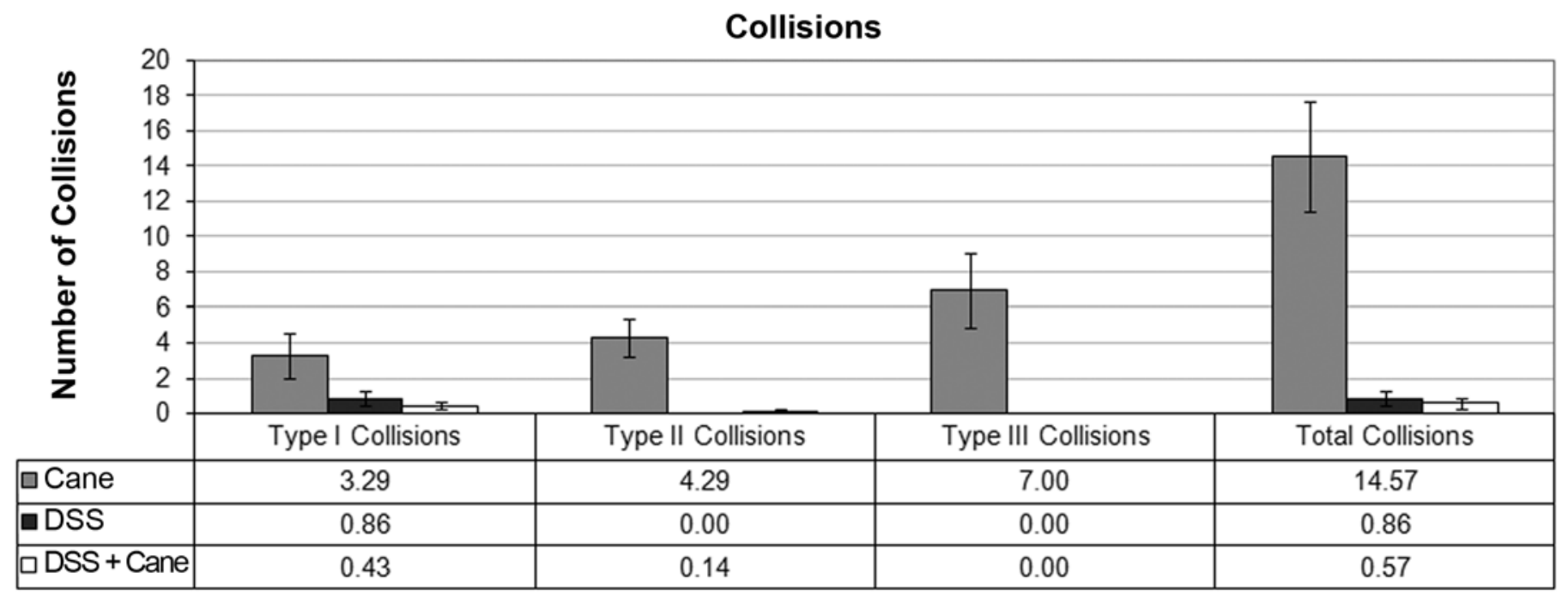

Figure 5.

Collisions. DSS = Drive-Safe System . 
Table 2.

Drive-Safe System (DSS) study results (mean \pm standard deviation).

\begin{tabular}{|c|c|c|c|}
\hline Measure & DSS & Cane & DSS + Cane \\
\hline \multicolumn{4}{|l|}{ NCT } \\
\hline Type I & $0.86 \pm 1.07$ & $3.29 \pm 3.30$ & $0.86 \pm 1.07$ \\
\hline Type II & $0.00 \pm 0.00$ & $4.29 \pm 2.87$ & $0.14 \pm 0.38$ \\
\hline Type III & $0.00 \pm 0.00$ & $7.00 \pm 2.33$ & $0.00 \pm 0.00$ \\
\hline Total & $0.86 \pm 1.07$ & $14.57 \pm 8.30$ & $0.57 \pm 0.79$ \\
\hline TCT (s) & $80.88 \pm 13.30$ & $65.71 \pm 23.08$ & $100.02 \pm 15.23$ \\
\hline PD & $0.10 \pm 0.19$ & $1.64 \pm 1.33$ & $0.43 \pm 0.51$ \\
\hline $\mathrm{E}$ & $2.56 \pm 1.99$ & $2.99 \pm 2.15$ & $2.71 \pm 1.97$ \\
\hline $\mathrm{F}$ & $0.96 \pm 1.65$ & $2.22 \pm 2.34$ & $1.50 \pm 1.98$ \\
\hline TWL & $9.30 \pm 3.59$ & $12.82 \pm 4.45$ & $10.72 \pm 5.00$ \\
\hline
\end{tabular}

\section{Total Collisions}

Table 2 shows the mean \pm SD total collisions (NCTT) for all three conditions. NCT-T was normally distributed. The Cane condition had significantly greater NCT$\mathrm{T}$ than the DSS condition $(p=0.02)$ and the DSS + Cane condition $(p=0.02)$. NCT-T did not significantly differ between the DSS and DSS + Cane conditions $(p>0.99)$.

\section{Task Completion Time}

Table 2 shows the mean \pm SD TCT for all three conditions. A Shapiro-Wilk test showed that TCT was normally distributed for all three conditions. Figure 6 shows the TCTs across the three experimental conditions. TCT did significantly differ between the Cane and the DSS conditions $(p=0.18)$ or the DSS and the DSS + Cane conditions $(p=0.70)$. TCT for the Cane condition was significantly lower than for the DSS + Cane condition $(p=$ 0.001 ).

\section{National Aeronautics and Space Administration-Task Load Index}

Figure 7 shows the subjective workload ratings across experimental conditions.

\section{Mental Demand}

Table 2 shows the mean \pm SD TLX-MD for all three conditions. A Shapiro-Wilk test showed that TLX-MD was normally distributed in all three conditions. TLXMD did not significantly differ between conditions $(F(2$, 12) $=0.761, p=0.49$.

\section{Physical Demand}

Table 2 shows the mean \pm SD TLX-PD for all three conditions. A Shapiro-Wilk test showed that TLX-PD was not normally distributed under the DSS condition $(p=0.006)$ but was normally distributed under the Cane and DSS + Cane conditions (Cane: $p=0.25$; DSS + Cane: $p=0.08$ ). Mean TLX-PD was significantly higher for the Cane condition than for the DSS condition $(Z=2.375, p=$ 0.02). Mean TLX-PD did not significantly differ between the Cane and DSS + Cane conditions $(Z=0.169, p=0.87)$ and between the DSS and the DSS + Cane condition $(Z=$ 1.577, $p=0.11$ ).

\section{Perceived Effort}

Table 2 shows the mean \pm SD TLX-E for all three conditions. A Shapiro-Wilk test showed that TLX-E was normally distributed under the Cane and the DSS conditions (Cane: $p=0.47$; DSS: $p=0.07$ ) but was not normally distributed under the DSS + Cane condition (DSS + Cane: $p=0.001)$. There was no statistically significant difference between the three conditions $\left(\chi^{2}(2, N=7)=\right.$ $0.286, p=0.87$ ).

\section{Frustration}

Table 2 shows the mean \pm SD TLX-F for all three conditions. A Shapiro-Wilk test showed that TLX-F was normally distributed in the Cane and the DSS + Cane conditions (Cane: $p=0.08$; DSS + Cane: $p=0.05$ ) but not in the DSS condition (DSS: $p<0.001$ ). There was no significant difference between conditions $\left(\chi^{2}(2, N=7)=\right.$ 3.714, $p=0.16)$. 


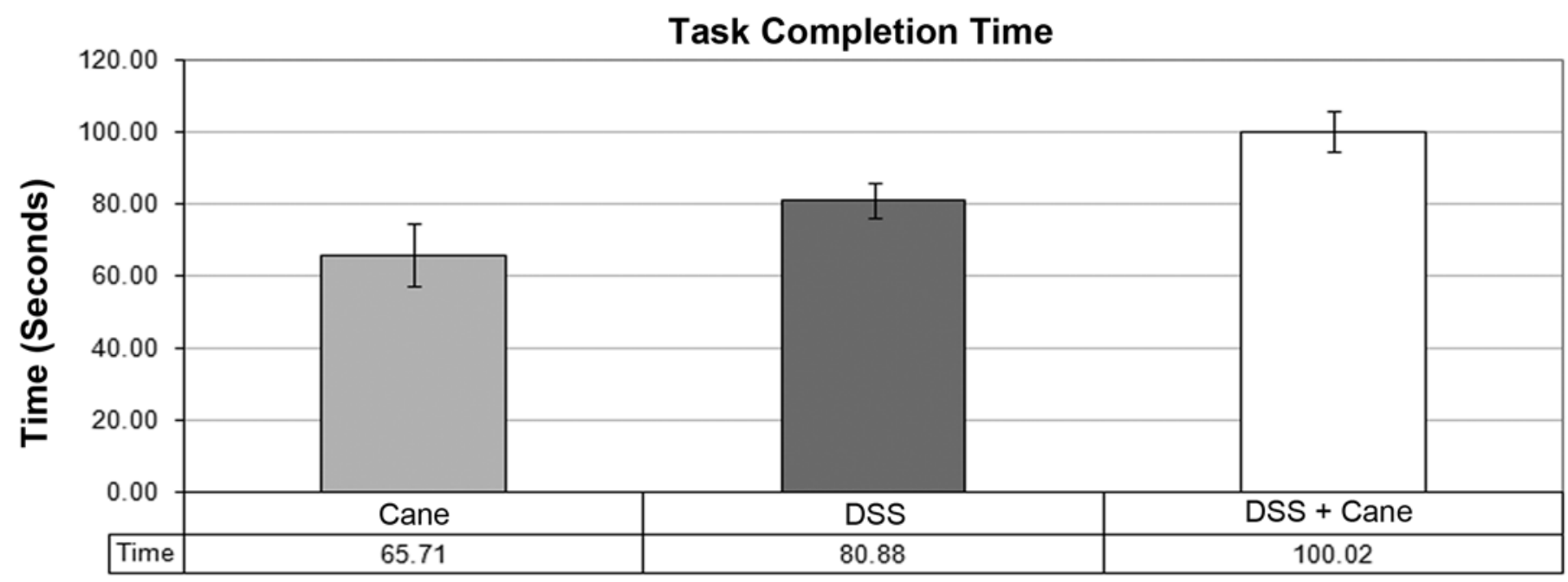

Figure 6.

Task completion time. DSS = Drive-Safe System.

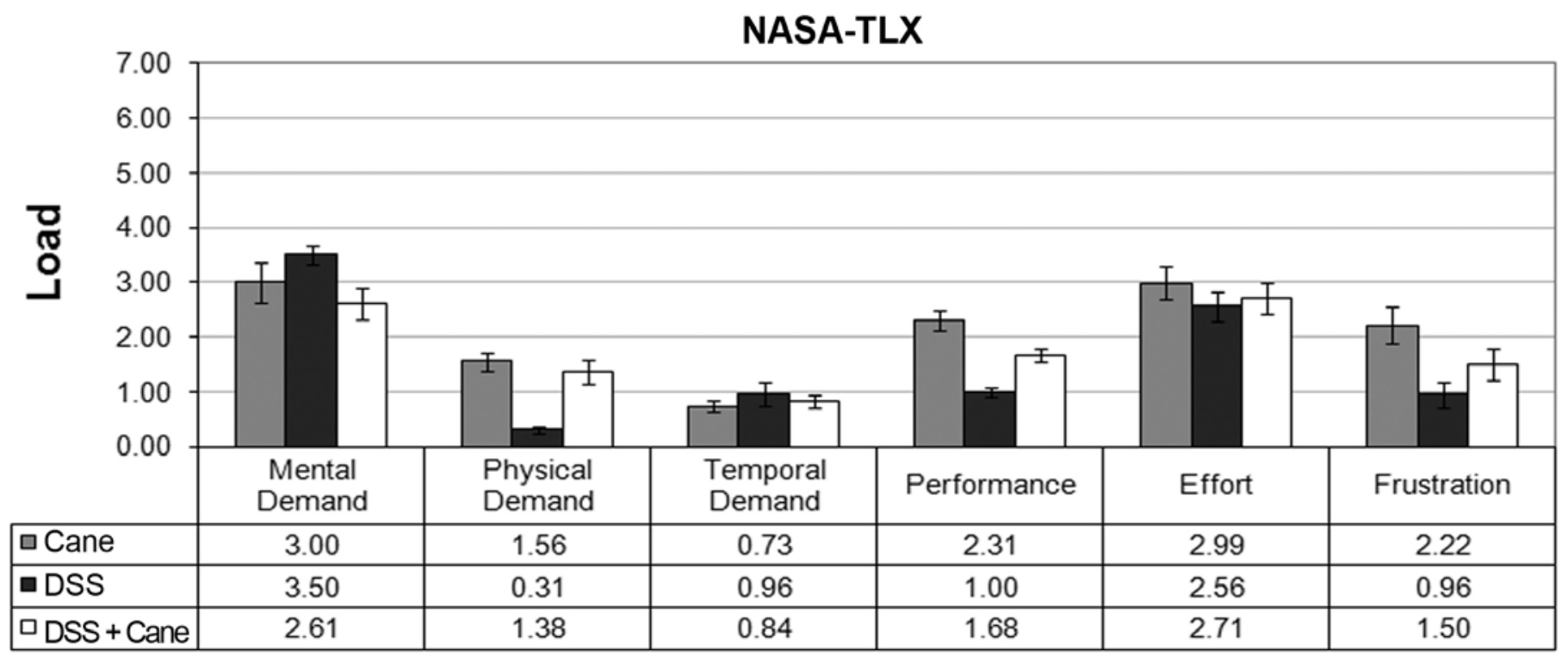

Figure 7.

National Aeronautics and Space Administration-Task Load Index (NASA-TLX) subjective workload. DSS = Drive-Safe System.

\section{Total Workload}

Table 2 shows the mean \pm SD TLX-TWL for all three conditions. A Shapiro-Wilk test showed that TLXTWL was normally distributed for all three conditions. TLX-TWL was not significantly different between conditions $(F(2,12)=2.160, p=0.16)$.

\section{DISCUSSION}

\section{Collisions}

Participants had significantly less total collisions when using DSS for navigation assistance than when using the cane alone. The collisions that did occur when 
using the DSS were typically of very low severity (mostly Type I) and could be avoided in the future by changing the programmed wheelchair stop thresholds in the DSS software. In contrast, the collisions that occurred when using the cane alone were more severe (mostly Type II and Type III). Participants in this study had expertise in using a cane and were skilled in navigating without visual cues. However, participants had very little experience using a power wheelchair, which may account for the large number of severe (Type II and Type III) collisions that occurred when participants were using the cane alone. Severe collisions when using the cane occurred because participants could not maintain the coordination between scanning speed and speed of the wheelchair. In many cases, participants were able to detect the obstacles but could not stop the wheelchair in time and had collisions. Further, not having any understanding of the wheelchair's dynamics and size also led participants to incorrectly judge the wheelchair's direction of movement, which caused collisions. On many occasions, the obstacle-scanning area with the cane was not wide enough and participants were unable to detect the obstacles even though they were in the direction of the wheelchair trajectory.

In the DSS + Cane condition, participants were required to use only the DSS for navigation, and once stopped by the DSS, they could use the cane to find the position of the obstacle and which direction was clear for the wheelchair's movement. Participants were instructed not to use the cane and the DSS at the same time, since the cane can move in front of the sensors, obstructing them and stopping the wheelchair. We did not collect information as to how long participants scanned with the cane and how long they used the DSS assistance in the DSS + Cane condition.

\section{Task Completion Time}

TCT was lower when using the cane than the DSS alone, but this difference was not significant. Lower TCT with the cane was achieved at the expense of hitting significantly more obstacles, as shown in Figure 8.

Participants in this study had expertise using a cane for navigation assistance in their everyday lives. This expertise was primarily responsible for their lower TCTs with the cane alone. The average speed of the wheelchair when using the DSS was lower than when using the cane alone, because the DSS slowed the wheelchair in the presence of obstacles. On a few occasions, the DSS stopped the wheelchair as a result of false-positive obstacle detection by the DSS, which added more time. The false-positive obstacle detection occurred because of certain architectural features of the testing area, which caused the reflection of ultrasound waves from one sensor to be picked up by the other sonar sensor. This phenomenon is very common with multiple sonar ranging and is termed "sensor cross talk." Future modifications of

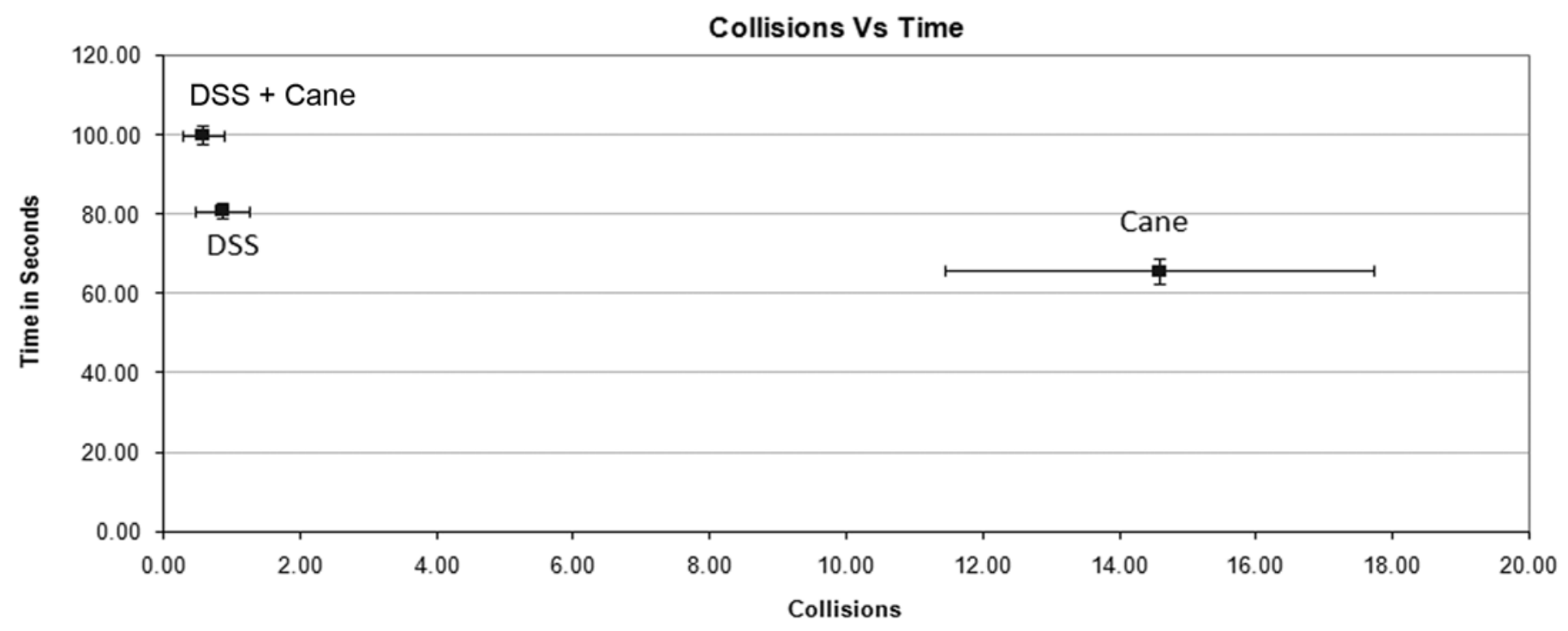

Figure 8.

Collisions versus time. DSS = Drive-Safe System. 
the DSS will require different ranging patterns of the sonar sensors to avoid the sensor cross talk. TCT was greater in the DSS + Cane condition because participants spent more time retrieving the cane to locate the position of obstacles around the wheelchair whenever they were stopped by the DSS.

In this study, navigation when using the DSS was safer but was achieved at the expense of longer TCT. Previously published studies with healthcare professionals and wheelchair users [30] underscore the relatively greater importance of safe navigation and collision avoidance in comparison with the time required to complete a given task.

\section{Physical Demand}

Physical demand was significantly higher in the Cane condition than the DSS condition. Physical demand when using the cane alone resulted from continuous scanning of the environment with the cane. However, in the DSS + Cane condition, participants did not need to scan the environment continuously because they relied on the DSS for obstacle detection and the cane was used only to find obstacle-free areas once the wheelchair was stopped by the DSS. When using the DSS alone, participants felt the lowest physical demand and this lower demand was mostly associated with the maneuvering of the joystick. Note, however, that physical demand was still low (1.56 on a scale from 0 to 7) under the cane condition, so whether physical demand was actually problematic is unclear.

\section{Mental Demand}

Participants did not experience differences in mental demand across experimental conditions, although that mental demand was relatively high in all three conditions. Participants in this study had never used the cane and/or the DSS with the power wheelchair before, so they had to learn to use both of these devices for navigation assistance, which may have been the reason for high mental demand.

\section{Frustration}

Frustration did significantly differ between conditions. Using a cane caused frustration because participants felt insecure about hitting obstacles, and even after trying hard, they could not prevent the collisions when using the cane alone. Frustration with the DSS was mainly caused by the repeated maneuvers required to steer the wheelchair around the obstacles, but these maneuvers were decreased as participants learned to use the DSS. Cross talk between ultrasonic rangefinders (URs) occurs when transmitted ultrasound waves from one UR are picked up by other UR and the second UR reports an incorrect range. The main cause of the cross talk is that all the URs in the DSS operate at the same frequency (42 KHz); therefore, it is hard for URs to distinguish whether the reflected ultrasound wave is coming from their transmission or some other UR's transmission.

Even though the direction and timing of ranging of each UR in the DSS is set in such a way as to avoid the cross talk, the high detection range of the UR (MaxSonarEZ series, MaxBotix; Brainerd, Minnesota) causes multiple reflections in a few geographic situations and these reflected waves are picked up by other URs, which then report false-positive obstacles and cause the wheelchair to stop itself. False-positive stops because of the UR cross talk perplexed participants and caused frustration and confusion. There are two main ways to reduce the sensor cross talk:

1. Change the ranging pattern of the URs to sequential ranging so no two URs will fire in the same direction at a given time.

2. Reduce the sampling rate of the URs such that enough time will lag between sequential firing of the two URs pointing in the same direction and no residual ultrasound will be in the air from the previous UR.

Note, however, that frustration was still low under both the conditions (Cane and DSS), so whether frustration was actually problematic is unclear.

Participants received auditory feedback from the DSS to help localize the position of the obstacles whenever the DSS stopped the movement of the wheelchair; however, many participants in the study had difficulty locating the position of the obstacles based on the auditory feedback. The DSS provides auditory feedback from five sensor nodes (only one sensor node at a time) to show the position of the obstacles in any of five directions (front right, front left, right, left, back). Since the feedback patterns from all five sensor nodes were similar, participants had difficulty determining which sensor node was providing the auditory feedback. In addition, the sound of the feedback was not loud enough, so it was hard for participants to hear the feedback. Good, intuitive auditory feedback can help drivers locate the obstacles and steer the wheelchair around them, without the need for multiple joystick maneuvers. Auditory feedback from 
the DSS can be improved by providing distinct and louder auditory feedback from each sensor node.

Auditory feedback is not always the most desirable choice for many people with disabilities. First, the continuous beeping from the DSS may cause disturbance in quiet environments. Second, many people with disabilities may not desire the unwanted attention to their disability that the auditory feedback might bring. Third, the feedback may not be heard or noticed by the user in open and/or crowded environments. Many participants suggested incorporating tactile feedback into the DSS. Tactile feedback will be more subtle and will not attract the attention of people other than users. Although this will be challenging to implement, future versions of the DSS should include tactile feedback so that people with hearing impairments, which are common in the aging population, can also benefit from the DSS. We also plan to have the option of audiofeedback through headphones in future versions of the DSS to ensure that the user will be able to hear the audiofeedback against background noise and so the system's beeping will not create a disturbance in quiet places.

\section{Perceived Effort}

No significant difference in perceived effort was found between conditions. Perceived effort was high in all three conditions, however, indicating that subjects felt they exerted noticeable effort in all three conditions. A noticeable trend occurred in perceived effort in the three conditions, but the results did not reach the significance level because of the small sample size.

Participants had to put extra physical effort into scanning of the environment by cane. Further, mental effort was associated with the use of the cane in learning to coordinate both hands while navigating toward the target sound. Mental effort when using the cane is likely to decline as people learn to coordinate both hands (scanning and maneuvering). During use of the DSS, it was the mental component that mainly contributed to the perceived effort because participants had difficulty estimating the position of obstacles while relying only on the auditory feedback. This caused participants to make multiple joystick maneuvers to move the wheelchair around an obstacle. The mental component in perceived effort while using the DSS along with a cane was reduced because participants were able to use the cane to locate the position and size of the obstacles and were able to reduce the number of joystick maneuvers required to move the wheelchair around an obstacle.

\section{Total Workload}

The hypothesis that total workload would be greater when using the cane alone was not supported. No significant difference was found between conditions. A noticeable trend occurred in TWL in the three conditions but the results did not reach the significance level because of the small sample size. When only the cane was used, TLX-MD and TLX-E together were responsible for 47 percent of TLX-TWL. Further, TLX-F accounted for 17 percent and TLX-PD 12 percent of TLX-TWL. When only the DSS was used, TLX-MD and TLX-E together were responsible for 65 percent of TLX-TWL. Further, TLX-F accounted for 10 percent and TLX-PD 3 percent of TLX-TWL. When the DSS along with the cane was used, TLX-MD and TLX-E together were responsible for 50 percent of TLX-TWL. Further, TLX-F accounted for 14 percent and TLX-PD contributed 13 percent of TLX-TWL.

A major advantage of the DSS for this population is that it can help users detect dynamic obstacles such as persons, pets, and vehicles. Visually impaired individuals are known to be adept at learning their environments and adapting to them in a short time. This ability would have introduced a confound in our data had we tested the DSS in a more naturalistic environment such as a home or workplace. Our primary goal in this phase of development and evaluation of the DSS was to evaluate the robustness of the hardware and software in detecting obstacles and providing valuable feedback for navigation assistance. Therefore, we decided to focus on a relatively simple setup for our experiment as opposed to a more naturalistic environment such as a home or a workplace. This allowed us to dynamically change the positioning of obstacles in order to test the capability of the DSS to detect dynamic obstacles. By providing the destination goal in the form of auditory cues, changing the obstacle positioning, and changing the participants' initial position across trials, we could ensure that they did not remember the path. This would not have been feasible to do in a more naturalistic environment.

We intend to modify the behavior of the DSS based on the findings of this experiment and later conduct more rigorous field trials with the modified version of the DSS using a single-subject design in more realistic settings such as a potential user's home or work environment. We 
believe that even in naturalistic settings, users will be less prone to hitting obstacles when using the DSS than when using a cane for navigation. Further, we hypothesize that people will be encouraged to explore newer environments when using the DSS.

\section{CONCLUSIONS}

Participants with visual impairments did not experience any significant difference in mental demand, perceived effort, frustration, or total workload. Participants felt less physical demand when driving the wheelchair while receiving navigation assistance from the DSS. "These results indicate that the DSS was able to provide reliable sensor coverage around the wheelchair and was able to avoid any catastrophic collisions which otherwise would have resulted in injury to the user or property damage." The DSS reduced the number of collisions and the severity of collisions but did not increase the time required to complete navigation tasks. Many results from this study showed differences in the dependent variables, but these differences did not reach significance because of the small sample size.

"One limitation of the research described above is that it was conducted in a controlled laboratory environment, which does not perfectly represent real world scenarios that people with disabilities encounter in their dayto-day lives." Results from this controlled environment study cannot be generalized to use of the DSS with the targeted nonambulatory, visually impaired individuals in real world scenarios; hence more research using "field trials" in which the DSS is used by nonambulatory, visually impaired individuals for extended periods of time in their naturalistic living spaces is necessary. Another advantage of field trials is that users will be able to experience the DSS for several hours and will provide valuable feedback regarding the performance of the DSS in unconstrained environments. "Investigators involved in the evaluation of the DSS in real world settings should also evaluate the users' ability to function when using the navigation assistance from the DSS using instruments such as FEW [34] or Power Mobility Indoor/Community Driving Assessment [11].”

When an individual obtains a new power wheelchair, he or she often purchases the wheelchair through a clinician, who is responsible for configuring the wheelchair (e.g., input method, maximum velocity, and maximum acceleration), selecting seating and positioning hardware (e.g., cushions, lateral supports, and headrests), and mounting other equipment on the wheelchair (e.g., communication devices, lap trays, and ventilators). The assessment process typically involves reviewing the client's medical history; measuring the client's flexibility and range of motion; assessing the perceptual, motor, and cognitive skills required to operate a power wheelchair; interviewing the client and caregivers; and, ultimately, some experimenting to identify a system that best meets the client's needs. Future evaluation of the DSS should involve occupational therapists, rehabilitation technologists, and wheelchair suppliers who provide wheelchair seating and mobility services. These participants should complete mock powered mobility assessments involving the DSS to evaluate the system's ability to coexist with standard seating and positioning hardware. In mock powered mobility assessment, the clinician will be asked to attach the DSS to a standard power wheelchair, along with whatever seating and positioning hardware is required to accommodate the needs of a hypothetical client, and to configure both the underlying power wheelchair and the DSS appropriately for the client. The clients will be assigned randomly from a set of hypothetical clients created by investigators based on their clinical experience with nonambulatory, visually impaired individuals. During the assessment, adult-sized and child-sized mannequins will be used to simulate the clients' bodies. The trials will also provide insight into the training and documentation that clinicians will need to effectively utilize the DSS in practice. The mock powered mobility assessments will be used to evaluate the effectiveness of the instructional materials and configuration software of the DSS.

Another limitation of the work described concerns the obstacles used. "Five foot high cylindrical cardboard tubes were used in this study as obstacles. The height and shape of these obstacles made them easy to be detected by sonar and infrared sensors and this is likely to have enhanced the obstacle detection performance of the DSS.”a

The safety of the wheelchair driver and safety of the environment are key factors that clinicians and rehabilitation practitioners consider when prescribing powered mobility for people with disabilities. The DSS's performance in avoiding collisions without significantly increasing TCT presents it as an encouraging intervention for safe and independent mobility for visually impaired wheelchair users. 


\section{ACKNOWLEDGMENTS}

\section{Author Contributions:}

Study concept and design: E. F. LoPresti, R. C. Simpson, V. Sharma. Acquisition of data: V. Sharma, R. C. Simpson.

Analysis and interpretation of data: V. Sharma, R. C. Simpson.

Drafting of manuscript: V. Sharma.

Critical revision of manuscript for important intellectual content:

V. Sharma, R. C. Simpson.

Statistical analysis: V. Sharma.

Obtained funding: E. F. LoPresti, R. C. Simpson.

Administrative, technical, or material support: V. Sharma,

E. F. LoPresti.

Study supervision: M. Schmeler, R. C. Simpson.

Financial Disclosures: Dr. LoPresti is the owner of AT Sciences, LLC. This study, funded by a National Institutes of Health Small Business Innovation Research grant, evaluates technology developed by AT Sciences for eventual commercialization.

Funding/Support: This material was based on work supported by a Small Business Innovation Research grant from the National Institutes of Health, National Institute of Child Health and Human Development (5R44HD040023-03).

Additional Contributions: Tara Minkus assisted Dr. Sharma with data collection. Dr. Sharma is now with the Department of Psychiatry, University of Pittsburgh, Pittsburgh, Pennsylvania.

Institutional Review: The study protocol was approved by the institutional review board of the University of Pittsburgh. All participants were read and signed informed consent forms.

Participant Follow-Up: The authors do not plan to inform participants of the publication of this study.

\section{NOTE}

${ }^{a}$ Quote from Sharma V, Simpson R, LoPresti E, Schmeler M. Evaluation of semiautonomous navigation assistance system for power wheelchairs with blindfolded nondisabled individuals. J Rehabil Res Dev. 2010;47(9):877-90.

PMID: 21174252

http://dx.doi.org/10.1682/JRRD.2010.02.0012

\section{REFERENCES}

1. Rosenbloom L. Consequences of impaired movement: A hypothesis and review. In: Holt KS, editor. Movement and child development. Philadelphia (PA): Lippincott; 1975.

2. Wright BA. Physical disability: A psychosocial approach. New York (NY): Harper \& Row; 1983.

http://dx.doi.org/10.1037/10589-000

3. Butler C. Effects of powered mobility on self-initiated behaviors of very young children with locomotor disability. Dev Med Child Neurol. 1986;28(3):325-32.
PMID: 2941328

http://dx.doi.org/10.1111/j.1469-8749.1986.tb03881.x

4. Erickson W, Lee C. 2007 disability status report: United States. Ithaca (NY): Cornell University Rehabilitation Research and Training Center on Disability Demographics and Statistics; 2008.

5. LaPlante MP, Kaye HS. Demographics and trends in wheeled mobility equipment use and accessibility in the community. Assist Technol. 2010;22(1):3-17. PMID: 20402043 http://dx.doi.org/10.1080/10400430903501413

6. Trefler E, Fitzgerald SG, Hobson DA, Bursick T, Joseph R. Outcomes of wheelchair systems intervention with residents of long-term care facilities. Assist Technol. 2004; 16(1):18-27. PMID: 15357146 http://dx.doi.org/10.1080/10400435.2004.10132071

7. Brandt A, Iwarsson S, Ståhle A. Older people's use of powered wheelchairs for activity and participation. J Rehabil Med. 2004;36(2):70-77. PMID: 15180221

8. Guiding blind people who are wheelchair users. London (England): Royal National Institute for the Blind; 2002.

9. Pranghofer M. Wheels and white canes: Tips for helping blind wheelchair users. Braille Monitor. Baltimore (MD): National Federation for the Blind; 1996.

10. Fehr L, Langbein WE, Skaar SB. Adequacy of power wheelchair control interfaces for persons with severe disabilities: A clinical survey. J Rehabil Res Dev. 2000;37(3): 353-60. PMID: 10917267

11. Hall K, Partnoy J, Tenenbaum S, Dawson DR. Power mobility driving training for seniors: A pilot study. Assist Technol. 2005;17(1):47-56. PMID: 16121645 http://dx.doi.org/10.1080/10400435.2005.10132095

12. Marshall S. Wheelchair rider injuries: Causes and consequences for wheelchair design and selection. J Rehabil Res Dev. 1997;34(2):vi. PMID: 9108340

13. Simpson RC. Smart wheelchairs: A literature review. J Rehabil Res Dev. 2005;42(4):423-36. PMID: 16320139 http://dx.doi.org/10.1682/JRRD.2004.08.0101

14. Ganoza D. Can a veteran with complete blindness drive a power wheelchair? Proceedings of the 25th International Seating Symposium; 2009 Mar 11-15; Orlando, FL. Pittsburgh (PA): Department of Rehabilitation Science and Technology, University of Pittsburgh. p. 193.

15. Greenbaum MG, Fernandes S, Wainapel SF. Use of a motorized wheelchair in conjunction with a guide dog for the legally blind and physically disabled. Arch Phys Med Rehabil. 1998;79(2):216-17. PMID: 9474006 http://dx.doi.org/10.1016/S0003-9993(98)90302-1

16. Simpson RC, LoPresti EF, Cooper RA. How many people would benefit from a smart wheelchair? J Rehabil Res Dev. 2008;45(1):53-71. PMID: 18566926 http://dx.doi.org/10.1682/JRRD.2007.01.0015

17. Kelly D. The enhancement of mobility for individuals who are both physically and visually disabled. Proceedings of 
the RESNA'99 Annual Conference: Spotlight on Technology; 1999 Jun 25-29; Long Beach, CA. Arlington (VA): RESNA Press; 1999. p. 227-29.

18. Simpson R, LoPresti E, Hayashi S, Guo S, Frisch R, Martin A, Ammer W, Ding D, Cooper R. The smart power assistance module for manual wheelchairs. Proceedings of the 2nd Cambridge Workshop on Universal Access and Assistive Technology; 2004 Mar 22-24; Cambridge, UK.

19. Simpson R, LoPresti E, Hayashi S, Guo S, Ding D, Ammer W, Sharma V, Cooper R. A prototype power assist wheelchair that provides for obstacle detection and avoidance for those with visual impairments. J Neuroeng Rehabil. 2005; 2:30. PMID: 16202136 http://dx.doi.org/10.1186/1743-0003-2-30

20. Simpson R, LoPresti E, Hayashi S, Nourbakhsh I, Miller D. The smart wheelchair component system. J Rehabil Res Dev. 2004;41(3B):429-42. PMID: 15543461 http://dx.doi.org/10.1682/JRRD.2003.03.0032

21. Simpson RC, Poirot D, Baxter F. The Hephaestus Smart Wheelchair System. IEEE Trans Neural Syst Rehabil Eng. 2002;10(2):118-22. PMID: 12236449 http://dx.doi.org/10.1109/TNSRE.2002.1031980

22. LoPresti EF, Simpson RC, Miller D, Nourbakhsh I. Evaluation of sensors for a smart wheelchair. Proceedings of the RESNA 2002 Annual Conference; 2002 Jun; Minneapolis, MN. Arlington (VA): RESNA Press.

23. Levine SP, Bell DA, Jaros LA, Simpson RC, Koren Y, Borenstein J. The NavChair Assistive Wheelchair Navigation System. IEEE Trans Rehablil Eng. 1999;7(4):443-51. PMID: 10609632 http://dx.doi.org/10.1109/86.808948

24. LoPresti EF, Sharma V, Simpson RC, Mostowy LC. Performance testing of collision-avoidance system for power wheelchairs. J Rehabil Res Dev. 2011;48(5):529-44. PMID: 21674403 http://dx.doi.org/10.1682/JRRD.2010.01.0008

25. Friedman A, Brown NR. Reasoning about geography. J Exp Psychol Gen. 2000;129(2):193-219. PMID: 10868334 http://dx.doi.org/10.1037/0096-3445.129.2.193

26. Cooper RA, Thorman T, Cooper R, Dvorznak MJ, Fitzgerald SG, Ammer W, Song-Feng G, Boninger ML. Driving characteristics of electric-powered wheelchair users: How far, fast, and often do people drive? Arch Phys Med Rehabil. 2002;83(2):250-55. PMID: 11833031 http://dx.doi.org/10.1053/apmr.2002.28020

27. Sharma V, Simpson R, LoPresti E, Schmeler M. Evaluation of semiautonomous navigation assistance system for power wheelchairs with blindfolded nondisabled individuals. J Rehabil Res Dev. 2010;47(9):877-90. PMID: 21174252 http://dx.doi.org/10.1682/JRRD.2010.02.0012
28. Hart SG. NASA-Task Load Index (NASA-TLX); 20 years later [Internet]. Moffett Field (CA): HFES; 2006. Available from: http:/humansystems.arc.nasa.gov/groups/TLX/downloads/ HFES_2006_Paper.pdf

29. Noyes JM, Bruneau DP. A self-analysis of the NASA-TLX workload measure. Ergonomics. 2007;50(4):514-19.

PMID: 17575712

http://dx.doi.org/10.1080/00140130701235232

30. Xiao YM, Wang ZM, Wang MZ, Lan YJ. [The appraisal of reliability and vitality of subjective workload assessment technique and NASA-Task Load Index]. Zhonghua Lao Dong Wei Sheng Zhi Ye Bing Za Zhi. 2005;23(3):178-81. Chinese. PMID: 16124892

31. Demers L, Monette M, Lapierre Y, Arnold DL, Wolfson C. Reliability, validity, and applicability of the Quebec User Evaluation of Satisfaction with assistive Technology (QUEST 2.0) for adults with multiple sclerosis. Disabil Rehabil. 2002;24(1-3):21-30. PMID: 11827151 http://dx.doi.org/10.1080/09638280110066352

32. Scherer MJ, Craddock G. Matching Person \& Technology (MPT) assessment. Rehabil Assist Technol. 2002;14(3): 125-31.

33. Mills T, Holm MB, Trefler E, Schmeler M, Fitzgerald S, Boninger M. Development and consumer validation of the Functional Evaluation in a Wheelchair (FEW) instrument. Disabil Rehabil. 2002;24(1-3):38-46. PMID: 11827153 http://dx.doi.org/10.1080/09638280110066334

34. Mills TL, Holm MB, Schmeler M. Test-retest reliability and cross validation of the Functioning Everyday with a Wheelchair instrument. Assist Technol. 2007;19(2):61-77. PMID: 17727074

http://dx.doi.org/10.1080/10400435.2007.10131866

Submitted for publication March 1, 2010. Accepted in revised form December 6, 2010.

This article and any supplementary material should be cited as follows:

Sharma V, Simpson RC, LoPresti EF, Schmeler M. Clinical evaluation of semiautonomous smart wheelchair architecture (Drive-Safe System) with visually impaired individuals. J Rehabil Res Dev. 2012;49(1):35-50. http://dx.doi.org/10.1682/JRRD.2010.03.0022

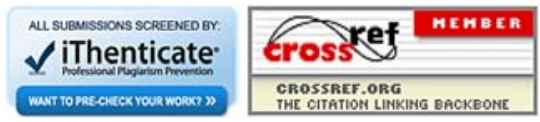


